\title{
Damage State Identification for Reinforced Concrete Columns in Uplift Due to Internal Building Detonations
}

\author{
* Lakshitha MG Wijesundara \\ BSc (Hons), MSc, PhD \\ Simon K Clubley \\ BEng(Hons) MBA PhD EurIng CEng MICE CITP MBCS \\ Infrastructure Research Group, \\ Faculty of Engineering and the Environment, University of Southampton \\ University Road, Southampton, SO17 1BJ, UK \\ Email: glk2g10@soton.ac.uk \\ Tel. +44 (0) 2380592862
}

Journal submission: November 2015 


\begin{abstract}
With the successful application of advanced computational fluid dynamics and structural response modelling techniques, we have conducted a set of comprehensive parametric studies on reinforced concrete column failure when subjected to high-rate, coupled uplift and shear typically induced by internal explosive blast. The outputs of the parametric studies have been processed to develop a generalised damage identification procedure for columns subjected to two main internal blast conditions. This paper describes the development of the damage identification procedure together with a number of worked examples illustrating its application and significance in practice.
\end{abstract}

Keywords: internal explosion; uplift force; damage assessment; dynamic response; blast; reinforced concrete; non-linear modelling

\title{
1. Introduction
}

As highlighted previously by the authors $[1,2]$, columns in internal blast environments are subjected to complex, high-rate axial and shear loads due to blast wave confinement and build-up pressures, leading to a noticeable loss of column structural integrity. With respect to the development of robust damage identification procedures for columns in such complex blast environments, it is essential to carefully take account of the effect of coupled, time-dependent uplift forces, because: (i) - these forces can significantly reduce the net axial compression force in columns, in some circumstances even leading to pure axial tension forces [1], and (ii) - the rebounding of adjacent floor plates and cross girders typically induce substantial axial compression forces [3] which can comfortably exceed the column ultimate design capacity. Nonetheless, the general literature on internal explosive blast and the effect of consequential complex loading conditions on the integrity of structural members currently remain in inconclusive. 
The existing literature in the fields of blast effects and protective structures contain research mainly focused on the design and performance assessment of structural systems subjected to external explosive blast [4-8]. In the context of damage state identification for columns, this means that columns have been comprehensively assessed only against two main loading conditions: pure transient-lateral blast loads or lateral blast loads coupled with static axial compression forces. The key aspects that have been of interest in these research include: (i) - the extent of localised damage, failure patterns and residual axial capacity of reinforced concrete $(R C)$ columns $[4,6]$ and, (ii) - the effect of extremely close-proximity or contact detonations on the response behaviour of $\mathrm{RC}$ columns $[5,8]$. In consistent with the nature of internal blast-induced loading conditions to a certain extent, multi-directional ground motions typically induced by seismic events can cause significant coupled, time-dependent axial and shear loads on columns [9]. As a consequence, the behaviour of RC columns subjected these time-dependent, coupled axial and shear loads has been of primary interest to many researchers $[10,11]$ leading to many design guidelines to account for the effects of axial force variation [12]. Nonetheless, there exists a fundamental problem with these guidelines and methods when applied to blast loading conditions due to the significant difference in loading rates. Blasts normally lead to significantly higher strain rates than those typical to seismic actions [13], thus causing highly-complex response and damage behaviours in RC members. As an example, RC components subjected to close-proximity, large-magnitudes explosions can exhibit an intense localised damage and fragmentation behaviour due to the direct transmission of high-intensity stress waves through the concrete, as observed by $[6,14-16]$. Conversely, there is also a beneficial effect of such high-rate loading conditions as they tend to enhance material strengths beyond the corresponding static limits.

A number of previous publications by the authors address the aspects key to the development of numerical modelling techniques [1] and data processing [2]. In particular, one of the recently- 
published articles [2] presents the research outputs in terms of a set of charts estimating the column residual axial capacity levels for a limited number of internal blast scenarios and mainly highlighting the influence of key input parameters on the overall extent of column damage. As an advancement on the previous publications, this paper presents a generalised damage identification procedure that can be applied to any specific assessment condition defined using column slenderness, concrete strength, reinforcement ratio and blast scenario. Section 2 of the paper summarises the research pertaining to the development of the fundamental numerical modelling procedure and its verification. The parametric study outputs key to the development of the damage identification procedure are discussed in section 3, which is followed by section 4 describing the developed assessment technique. Section 5 provides a set of worked examples. In section 6, conclusions are presented.

\section{Numerical modelling of internal blast and column response}

In this research, high-resolution CFD simulations of internal building detonation and wave propagation were conducted using the hydrocodes "Air3D" [17] and Autodyn [18]. The hydrocode Air3D is an explicit, finite volume formulation, which solves one-, two and three-dimensional forms of the Euler equations on a regular Cartesian grid using equally spaced, square, and cubic cells. Although the regular Cartesian mesh used in Air3D allows rapid one-dimensional sweeps of the flow solver in each of the three dimensions [17], the hydrocode fails in modelling the effect of close-proximity detonations due to: (i) - the influence of explosive substances on the magnitudes of loading, and (ii) - the effect of after-burn, which is an additional energy-release mechanism governed by further oxidation of detonation products as the shock wave propagates through the surrounding air. With respect to modelling this complex fluid-structure interaction behaviour typical to close-proximity detonations, Autodyn performs better in comparison to Air3D, thus it was used for modelling internal explosions associated with small scaled distances. 
In Autodyn, alternative numerical processors are available to model detonation and wave propagation through the air. In this research, the Eular processor that allows for multi-material flow capabilities was employed, with remapped data registries between different flow fields (see [1, 19] for more details). Although the single material Eular-Ideal-Gas flow solver is fast and efficient compared to other Eular processors in blast simulations, it was not adoptable in this research due to the lack of sufficient expansion of the explosives prior to remapping data registries. This can be explained by using the equations of state (EOS) used for modelling TNT and air, given in Eqs 1 [20] and 2 [18] respectively.

$$
P=C_{1}\left(1-\frac{\omega}{R_{1} V}\right) e^{-R_{1} V}+C_{2}\left(1-\frac{\omega}{R_{2} V}\right) e^{-R_{2} V}+\frac{\omega E}{V}
$$

Where $P$ is the hydrostatic pressure, $C_{1}, C_{2}, R_{1}, R_{2}$ and $\omega$ are empirically derived constants based on the type of explosives; $V$ is the specific volume of the detonation products and $E$ is specific internal energy.

$$
P=(\gamma-1) \rho E
$$

Where $P$ is the pressure, $\gamma$ is the adiabatic constant and is equal to $1.4, \rho$ is the air density and is equal to $1.225 \mathrm{~kg} / \mathrm{m}^{3}$ and $E$ is the specific air internal energy $\left(=2.068 \times 10^{5} \mathrm{~kJ} / \mathrm{kg}\right)$. Autodyn calculates the density of the detonation products, $\rho(E q-3)$ using the compression ratio, $\mu$. If the value of $\mu$ decreases below -0.99 , the density of the detonation products tends to be very small [21], and at this stage (at large volumetric ratios) the first two terms of the JWL EOS (Eq - 1) become negligible, converging the JWL EOS to that of an ideal gas $(\mathrm{Eq}-2)$ with $\gamma=1.3$ and $\rho=$ $1 \times 10^{-4} \mathrm{~g} / \mathrm{cm}^{3}$. To employ the Eular-Ideal-Gas floor solver for the $3 \mathrm{D}$ domain, the floor state conversion from JWL-EOS to ideal gas requires to occur prior to remapping the data from 1D (or 2D) analysis; however, this cannot always be achieved particularly in the case of considerably near-filed detonations such as internal explosive blasts. 


$$
\rho=\rho_{0}(1+\mu)
$$

To take account of the effect of after-burn and consequential time-dependent energy release, Autodyn provides a modification to the last term in $\mathrm{Eq}-1$ based on the approach proposed by Miller [22], as described by Eq-4.

$$
P=C_{1}\left(1-\frac{\omega}{R_{1} V}\right) e^{-R_{1} V}+C_{2}\left(1-\frac{\omega}{R_{2} V}\right) e^{-R_{2} V}+\frac{\omega(E+\lambda Q)}{V}
$$

where $d \lambda / d t=a(1-\lambda)^{m} P^{n} ; \mathrm{Q}$ is additional specific energy (see [23]); a is energy release constant; $m$ and $n$ are energy release exponent $(\sim 1 / 2)$ and pressure exponent $(\sim 1 / 6)$ respectively.

Figure 1 illustrates the wave propagation and fluid-structure interaction due to a detonation of $25 \mathrm{~kg}$ of TNT equivalence within a vented virtual building arrangement, where the blast wave is confined only between the ground and the upper floors whilst permitting to flow out along the building perimeter. The main aspects pertaining to CFD simulations (e.g. mesh sensitivity, remapping process and comparison of pressure and impulse histories obtained separately from Autodyn and Air3D simulations) are not of primary interest in this paper. The interested readers are directed to the previous publications by the authors $[1,2]$. To demonstrate CFD modelling accuracy, a comparison study was conducted using a set of ConWep [24] predictions in terms of peak reflected overpressure $\left(P_{r}\right)$, total reflected impulse $\left(I_{r}\right)$ and shock arrival time $\left(T_{a}\right)$ estimated for 30 different internal blast scenarios. Based on the standoff distance $(R)$ and TNT equivalence $(M)$, the ConWep predictions pertaining to the primary pressure pulse (see Figure 2) in each simulation were compared with corresponding CFD simulation results. These are provided in Table 1 together with the column internal forces (shear, $V$, peak axial force, $N$ and peak bending moment, M) estimated separately from applied element (AE) and finite element (FE) structural response modelling techniques. With respect to modelling internal blast, the results indicate a good overall consistency between CFD and ConWep predictions. The slight deviations of the CFD predictions 
compared to the ConWep results can be attributed to main simplifications used with CFD simulations, including: (i) - perfect ground reflection, (ii) - above-ground detonation (approximately $0.5 \mathrm{~m}$ above the ground), and (iii) - blast wave reflection at a finite column surface leading to a notable clearing effect.

The primary computer program used to model the transient-dynamic response of columns was ELS [25] which is based on the AE method [26] for modelling the response of structures subjected to extreme loading conditions such as blast and impact. The fundamental numerical modelling procedure, material models and verification studies (both qualitative and quantitative) have already been discussed in details in a number of previous publications by the authors $[1,27]$. As a further extension to the verification studies reported in the previous publications, this paper also provides a simplistic comparison of column internal forces estimated separately using the $A E$ and FE methods (see Table 1). The primary interest of this comparison was to ensure the integrity of AE models prior to conducting more comprehensive verification studies using experimental test data. In this comparison, although the reinforcement were modelled explicitly as unique structural components with interface (see Figure 3), material properties for the concrete and reinforcement were assumed to be linear-elastic for simplicity.

\section{Development of the damage state identification procedure}

The underlying procedure used for the development of this damage assessment procedure consists of two main steps: (i) - a comprehensive parametric study to develop a database of column response and damage patterns corresponding to different internal explosive blast scenarios, and (ii) - data processing (multi-variable, non-linear curve fitting analysis) and interpretation of results. Prior to the parametric study, input parameters key to the response and failure of columns were identified using a set of independent numerical simulations [19], namely: 
column slenderness properties (width, $B$; depth, $D$; height, $H$ ), blast scenario (TNT equivalence, $M$; standoff distance, $R$ ), reinforcement ratio (longitudinal, $\rho_{l}$; transverse, $\rho_{t}$ ) and design compressive strength of concrete, $f_{c k}$. In each analysis case, two output variables (damage index, $\eta$ and residual axial capacity index, $\alpha$ ) were derived based on the extent of overall column damage. As an example, Figure 4 illustrates the procedure used for estimating $\alpha$ using an incremental static, axial compression force applied to the column following the blast loading stage, until the column fails in direct compression. Referring to Figure $4, \alpha$ can be obtained by using the axial force versus axial shortening curves derived for both blast-induced damaged and undamaged conditions. In other words, $\alpha$ is defined as $N_{d} / N_{u d}$, where $N_{d}$ and $N_{u d}$ are the column axial compression capacity corresponding to damaged and undamaged conditions, respectively. As an illustration, the data given in Figure 4 can be used to estimate the values of $\alpha$ for the two damage conditions denoted as $B$ and $C$. These are equal to 0.79 and 0.68 respectively. The damage index $\eta$ is a qualitative parameter defined based on the key observations such as the extent of column overall damage and integrity of reinforcement. Table 2 provides part of the parametric study outputs pertaining to the two internal blast environments considered in this research: vented and contained. In the case of vented blast scenario, the building has neither strong perimeter walls nor partition walls (e.g. building arrangements typical to open-plan parking premises). Conversely, contained blast conditions are typical in explosive blast events occurring within areas such as basement parking or storage premises where the presence of non-frangible perimeter walls can completely reflect the entire blast wave, further increasing the magnitudes of blast loads on structural components.

The data processing step involves with multi-variable, non-linear curve fitting analyses using a Chisquare minimisation approach [28]. In this, the global regression function which relates the input variables to the damage index, $\eta$ takes the form as described by Eq -5 . The column slenderness input parameters $(B, D$ and $H)$ were shown to have polynomial relationships with $\eta$, whilst the 
relationships corresponding to other variables $\left(M, R, \rho_{l}\right.$ and $\left.\rho_{t}\right)$ were power functions (see [19] for further details).

$$
\begin{gathered}
\eta[f(\alpha)]=a\left(b+c \cdot[B]+d \cdot[B]^{2}\right) \times\left(e+f \cdot[D]+g \cdot[D]^{2}\right) \times\left(h+i \cdot[H]+j \cdot[H]^{2}\right) \\
\times\left(k+l \cdot[M]^{m}\right) \times\left(n+o \cdot \frac{1}{[R]^{2}}\right) \times\left(\frac{p}{10^{12}} \times \frac{1}{\left[\rho_{l}\right]^{q}}\right) \times\left(r-s \cdot\left[\rho_{t}\right]^{t}\right)
\end{gathered}
$$

The global non-linear, multiple regression function described by Eq -5 is the mathematical representation of a specific data set. Once the model parameters $(a, b, c, \ldots, t)$ are estimated, the function can be used to develop a graphical representation of the data set for repetitive application purpose. To derive the model parameters, curve fitting analyses were conducted for each data set corresponding to different compressive strength classes (see Table 2), separately for vented and contained blast conditions. As an example, Tables 3 and 4 provides the model parameters estimated using the data set for $f_{c k}=40 \mathrm{MPa}$ corresponding to the vented internal blast scenario. When performing nonlinear curve fitting, an iterative procedure is employed that minimises the reduced chi-square $\left(\chi^{2} r d\right)$ value to obtain the optimal model constant values. The $\chi^{2} r d$ value and adjusted coefficient of determination $\left(R^{2} a d\right)$ corresponding to the outputs provided in Tables 3 and 4 are 2.40 and 0.90 respectively, indicating a good fitness of data. The reduced chi-square was obtained by dividing the residual sum of squares (RSS) by the degrees of freedom (DOF). Although this is the quantity that is minimised in the iteration process, it is typically not a good measure to determine the goodness of fit [28]. On the other hand, coefficient of determination $\left(R^{2}\right)$ provides a better understanding of the fitness of data; however, a larger value of $R^{2}$ (closer to unity) does not necessarily mean a better fit because the degrees of freedom can also affect the value. As a consequence, if more parameters are introduced, the $R^{2}$ value will increase, but this does not imply a better fit. To overcome this problem, the adjusted coefficient of determination was estimated, as 
this parameter can take accounts for the degrees of freedom and leads to a better estimate of the goodness of fit.

The primary objective of the use of damage identification procedure is to identify the extent of column damage in terms of $\eta$ and $\alpha$. This requires a set of distinct damage classes that appropriately describe the extent of column damage with high practical relevance, because depending upon the input variable values, column overall damage can range from a no-damaged state to an imminent collapse state (complete compression failure or sheared-off). By carefully investigating the parametric study outputs, it was possible to define a damage classification system consisting of five damage classes as provided in Table 5. Table 5 also includes the appropriate range of each output variable $(\eta$ and $\alpha$ ) for each damage class. Figure 5 shows the relationships between $\eta$ and $\alpha$ derived separately for each damage class using the results.

\section{Damage identification procedure}

This section describes the underlying stepwise procedure developed for damage identification.

Step 1: The input variables $B, D, H, \rho_{l}$ and $\rho_{t}$ were fixed. For example, the normalised values of $B, D$ and $H$ (denoted respectively as $B^{\prime}, D^{\prime}$ and $H^{\prime}$ ) were $350 \mathrm{~mm}, 350 \mathrm{~mm}$ and $3 \mathrm{~m}$ respectively. Using these fixed values and the global non-linear regression function given by Eq - 5, a simplified equation that relates $M$ and $R$ to a new parameter termed 'normalised damage index $\left(K_{D}\right)^{\prime}$ ' was derived as given by Eq -6 , where $K_{1}, K_{2}, K_{3}, K_{4}$ and $K_{5}$ are constants that depend on the normalised input variables.

$$
K_{D}=K_{1} \times K_{2} \times K_{3} \times\left(k+l .[M]^{m}\right) \times\left(n+o \cdot \frac{1}{[R]^{2}}\right) \times K_{4} \times K_{5}
$$

Once the model constants $k, l, m, n$ and $o$ are obtained from curve fitting analyses, it is possible to interpret $K_{D}$ graphically in terms of different combinations of $M$ and $R$. Considering this, two 
separate sets of charts for vented and contained internal blast conditions were developed. For each blast condition, five charts were constructed based on the design compressive strength of concrete: 30MPa, 40MPa, 50MPa, 60MPa and 100MPa. These charts are shown in Figures 6(a)-(e) and 7(a)-(e) for vented and contained internal blast conditions respectively. Each chart also comprises a reference line $\left(z=0.3 \mathrm{~m} / \mathrm{kg}^{1 / 3}\right)$ beyond which the curves were extended purely on the basis of data extrapolation.

Step 2: The charts described in step 1 correspond with the normalised values of $B, D, H, \rho_{l}$ and $\rho_{t}$. As a consequence, modifications are necessary for actual values of the normalised input variables. In this step, modifications in terms of the correction factors $\beta_{B}, \beta_{D}$ and $\beta_{H}$ for column slenderness input parameters $B, D$ and $H$ are derived. Considering Eqs -5 and 6 , the following relationships can be written for $\beta_{B}, \beta_{D}$ and $\beta_{H}$ :

$$
\begin{gathered}
\beta_{B}=\frac{b+c \cdot[B]+d \cdot[B]^{2}}{b+c \cdot[350]+d \cdot[350]^{2}} \\
\beta_{D}=\frac{e+f \cdot[D]+g \cdot[D]^{2}}{e+f \cdot[350]+g \cdot[350]^{2}} \\
\beta_{H}=\frac{h+i \cdot[H]+j \cdot[H]^{2}}{h+i \cdot[3]+j \cdot[3]^{2}}
\end{gathered}
$$

Alternatively, the correction factors $\beta_{B}, \beta_{D}$ and $\beta_{H}$ can be obtained from the charts shown in Figures 8 and 9 for vented and contained internal blast conditions respectively. In either of the two methods, the correction factor for any column slenderness input parameter requires to converge to unity when the actual value is equal to its normalised value.

Step 3: Using a similar approach to that discussed in step 2, the correction factors for longitudinal and transvers reinforcement ratios $\beta_{\rho l}$ and $\beta_{\rho t}$ are derived in this step. These can be written as: 


$$
\begin{gathered}
\beta_{\rho l}=1.24 \times\left(\rho_{l}\right)^{-0.178} \\
\beta_{\rho t}=0.99-\frac{2.07}{10^{12}}\left(\rho_{t}\right)^{4.29}
\end{gathered}
$$

These relationships were derived using the global regression function ( $E q-5)$ and the model constants $p, q, r, s$ and $t$ estimated from the non-linear curve fitting analyses.

Step 4: This step involves with estimating the damage index $\eta$ using $K_{D}, \beta_{B}, \beta_{D}, \beta_{H}, \beta_{\rho l}$ and $\beta_{\rho t}$ estimated in the previous steps:

$$
\eta=K_{D} \times \beta_{B} \times \beta_{D} \times \beta_{H} \times \beta_{\rho l} \times \beta_{\rho t}
$$

The estimated damage index is subsequently used to obtain the appropriate damage class together with the column residual axial capacity using the grey scale shown in the damage identification chart for the desired compressive strength class and internal blast condition. For compressive strengths in between any two successive $f_{c k}$ values, $\eta$ can be estimated from interpolation; however, in this case, the appropriate damage class should be obtained from the chart corresponding to the smaller $f_{c k}$ value to ensure safe conservative predictions.

\section{Application of the proposed method}

This section discusses a set of illustrative examples for the application of the damage identification procedure described in section 4 . In the first part, the column dimensions $B, D$ and $H$ were defined to be $250 \mathrm{~mm}, 350 \mathrm{~mm}$ and $3.5 \mathrm{~m}$ respectively. The column was considered to have 8 longitudinal reinforcing bars of $25 \mathrm{~mm}$ diameter, and $10 \mathrm{~mm}$ diameter stirrups with a spacing of $100 \mathrm{~mm}$. The clear concrete cover was assumed to be $25 \mathrm{~mm}$. The blast scenario was defined at $60 \mathrm{~kg}$ of TNT equivalence detonated at a standoff distance of $2.5 \mathrm{~m}$. 
Once the blast scenario, column dimensions and reinforcement details are defined, the damage identification procedure can be used to predict the appropriate damage class (DC) and consequential column residual axial capacity. As an example, if the design compressive strength of concrete, $f_{c k}$, is assumed to be $40 \mathrm{MPa}$, the corresponding damage class for vented and contained internal blast conditions are 9.28 and 13.34 respectively. In both cases, the column remains in 'Moderate' damage class ( $8 \leq \eta \leq 16)$; however, the column residual axial capacity corresponding to the vented blast condition (78\%) decreases to $68 \%$ under the contained blast condition, column axial capacity drop of $10 \%$ purely due to further confinement of the blast wave. Table 6 provides the entire output data set including the values of $\eta$ and $\alpha$ together with the damage class corresponding to each $f_{c k}$ value, estimated separately for vented and contained internal blast conditions. It is evident from the results that the column when subjected to the vented internal blast will have reduced damage (LD) at $f_{c k}=50 \mathrm{MPa}$ or greater, compared to the damage extent typical to lower strength classes $(<40 \mathrm{MPa})$. Similarly, in the case of contained internal blast, the column exhibits a reduced damage level (LD) only at an $f_{c k}$ value in between 60MPa and 100MPa, clearly indicating the extent of increased damage due to the contained blast.

To further illustrate the application of the proposed method, it was used to estimate the value of $\eta$ corresponding to different TNT equivalences ranging from $10 \mathrm{~kg}$ to $150 \mathrm{~kg}$ (see Figure 10a). In this, the contained and vented blast conditions are denoted as ' $V$ ' and ' $C$ ' respectively. The problem input variables remained the same as described earlier, except the column's depth and width which were changed to define two assessment groups ( $C 1$ and $C 2$ ) with different column orientations with respect to the direction of the blast wave. In the assessment group ' $C 1^{\prime}, B$ and $D$ were $250 \mathrm{~mm}$ and $350 \mathrm{~mm}$ respectively, whilst those corresponding to the analysis group ' $\mathrm{C} 2^{\text {' were }}$ $350 \mathrm{~mm}$ and $250 \mathrm{~mm}$; a reduced column lateral stiffness in $C 2$ compared to that in $C 1$. Of the four assessment scenarios denoted in Figure 10 a as $C 1_{-} V, C 1_{-} C, C 2 \_V$ and $C 2 \_C, C 1 \_V$, in which case 
the column deforms about its major bending axis due to the effect of vented blast, indicates a high level of resistance against the blast. Conversely, when the column is considered to deform about its minor bending axis due to the effect of contained blast (assessment case C2_C), it indicates a noticeably low level of resistance against the blast, and the column also tends to exhibit a complete collapse at a TNT equivalence of $116 \mathrm{~kg}$ or greater. For the same assessment scenarios, Figure 10b shows the relationships between $\eta$ and $R$ (TNT equivalence was fixed at $60 \mathrm{~kg}$ ). It is evident from Figure $10 \mathrm{~b}$ that the column vulnerability to the blast (60kg of TNT) exponentially increases with decreasing standoff distance, $R$. At standoff distances equal to or smaller than approximately $1.5 \mathrm{~m}$, the column exhibits an imminent collapse in all assessment scenarios. To ensure that the column can survive the blast either in vented or contained wave propagation condition, the required safe standoff distance can be considered as $3.5 \mathrm{~m}$ (see Figure 10b); however the column can still be subjected to moderate damage as described in Table 5. The extent of acceptable damage level depends on many factors such as the overall protection level required for the building and the type of column retrofits available as means of blast protection measures.

\section{Conclusions}

\section{References}

[1] Wijesundara, L.M. and S.K. Clubley, Numerical modelling of reinforced concrete columns subject to coupled uplift and shear forces induced by internal explosions. Structure and Infrastructure Engineering, 2014, DOI: 10.1080/15732479.2014.1002502.

[2] Wijesundara, L.M. and S.K. Clubley, Residual Axial Capacity of Reinforced Concrete Columns Subject to Internal Building Detonations. International Journal of Structural Stability and Dynamics, 2015, DOI: 10.1142/S0219455415500509.

[3] FEMA, Design Guidance for Shelters and Safe Rooms, in Risk Management Series, 2006, Federal Emergency Management Agency [FEMA], Report No: 453.

[4] Fujikake, K. and P. Aemlaor, Damage of reinforced concrete columns under demolition blasting. Engineering Structures, 2013. 55: p. 116-125. 
[5] Roller, C., et al., Residual load capacity of exposed and hardened concrete columns under explosion loads. Engineering Structures, 2013. 55: p. 66-72.

[6] Wu, K., B. Li, and K. Tsai, Residual axial compression capacity of localized blast-damaged RC columns. International Journal of Impact Engineering, 2011. 38(1): p. 29-40.

[7] Astarlioglu, S., et al., Behavior of reinforced concrete columns under combined effects of axial and blast-induced transverse loads. Engineering Structures, 2013. 55: p. 26-34.

[8] Bao, X. and B. Li, Residual strength of blast damaged reinforced concrete columns. International Journal of Impact Engineering, 2010. 37(3): p. 295-308.

[9] Kim, S.J., C.J. Holub, and A.S. Elnashai, Analytical assessment of the effect of vertical earthquake motion on RC bridge piers. Journal of Structural Engineering, 2010. 137(2): p. 252260.

[10] Jeon, J.-S., et al., Damage assessment of older highway bridges subjected to threedimensional ground motions: Characterization of shear-axial force interaction on seismic fragilities. Engineering Structures, 2015. 87: p. 47-57.

[11] Kunnath, S.K., et al., Effect of near-fault vertical ground motions on seismic response of highway overcrossings. Journal of Bridge Engineering, 2008. 13(3): p. 282-290.

[12] Lee, D.H. and A.S. Elnashai, Inelastic seismic analysis of RC bridge piers including flexureshear-axial interaction. Structural Engineering and Mechanics, 2002. 13(3): p. 241-260.

[13] Ngo, T., et al., Blast loading and blast effects on structures-an overview. Electronic Journal of Structural Engineering, 2007. 7: p. 76-91.

[14] Zhou, X., et al., Numerical prediction of concrete slab response to blast loading. International Journal of Impact Engineering, 2008. 35(10): p. 1186-1200.

[15] Zineddin, M. and T. Krauthammer, Dynamic response and behavior of reinforced concrete slabs under impact loading. International Journal of Impact Engineering, 2007. 34(9): p. 15171534.

[16] Xu, K. and Y. Lu, Numerical simulation study of spallation in reinforced concrete plates subjected to blast loading. Computers \& structures, 2006. 84(5): p. 431-438.

[17] Rose, T.A., Air3D User's Guide. 7.0, 2003, Cranfield University: Cranfield.

[18] Century Dynamics, Theorical manual for Autodyn (Version 14.0), 2011, Ansys: Canonsburg, PA.

[19] Wijesundara, L.M., Design and Assessment of Reinforced Concrete Columns in Uplift Due to Internal Building Detonations, PhD Thesis, in Faculty of Engineering and the Environment. 2015, University of Southampton: Southampton, UK. p. 367.

[20] Lee, E.L., H.C. Hornig, and J.W. Kury, Adiabatic Expansion of High Explosive Detonation Products, 1968, Lawrence Radiation Laboratory, University of California, Report No: UCRL 50422.

[21] Sherkar, P., A.S. Whittaker, and A.J. Aref, Modeling the Effects of Detonations of High Explosives to Inform Blast-Resistant Design 2010, MCEER Thrust Area 3, Innovative Technologies, University of Buffalo, Report No: MCEER -10-0009.

[22] Miller, P.J., A Reactive Flow Model with Coupled Reaction Kinetics for Detonation and Combustion in Non-Ideal Explosives. Mat. Res. Soc. Symp. Proc., 1996. 418: p. 413-420.

[23] Cooper, P., Explosives Engineering. 1996, New York: Wiley-VCH. 
[24] Hyde, D., ConWep Conventional Weapons Effects Programme, 1992, U.S. Army Engineer Waterways Experiment Station Vicksburg, MS.

[25] Applied Science International [ASI], Theoretical Manual for Extreme Loading ${ }^{\circledR}$ for Structures (ELS), 2013: Durham, NC.

[26] Tagel-Din, H. and K. Meguro, Applied Element Method for Dynamic Large Deformation Analysis of Structures. Structural Eng./Earthquake Eng., International Journal of the Japan Society of Civil Engineers (JSCE), 2000. 17(2): p. 215-224.

[27] Wijesundara, L.M., S.K. Clubley, and M. Hahn. Non-linear Modelling of Reinforced Concrete Columns Subject to Internal Explosions and Uplift Effects: CFD and Applied Element Methods. in In the Proceedings of Structures Congress. 2014. Boston, MT: ASCE, DOI: 10.1061/9780784413357.174.

[28] OriginLab Corporation, Origin 8.1 User Guide, Data Analysis and Graphing Software, 2007, OriginLab Corporation Northampton, MA, USA. 